\title{
Value, the new medical mantra: misunderstood, abused, and underused
}

\author{
Lodovico Balducci
}

Received: 8 March 2013/Accepted: 8 March 2013/Published online: 26 March 2013

(C) Springer-Verlag Italia 2013

Nowadays it is difficult to attend a medical conference without a discussion of value $[1,2]$. This trend to make practitioners and institutions accountable for quality of care, patient satisfaction, and cost represents a historical shift in medical practice. It is nothing less than an attempt to deliver patient-centered care and to empower the patient to make the choice more congruent with his/her desires and expectations [3]. Our publication, the Journal of Medicine and the Person (JMP), is entitled to some credit for this new trend. Since the very beginning, under the leadership of Giancarlo Cesana and Marco Ferrario, the preoccupation of the journal has been to fulfill and promote the mission of our movement. This involves giving a voice to the patient as a person, making sure that the patient receives the treatment that best promises to restore his/her wholeness irrespective of the cost and of institutional and social barriers that may compromise treatment access. At the same time JMP has aimed to dissipate the confusion generated by an increasingly complex medical establishment in the minds of patients and practitioners alike. Thus we could not be happier to welcome this novel approach to medicine and we feel duty-bound to enter the discussion of value in medicine.

The accepted definition of value in medical care is the one proposed by Porter in a seminal article [4]: quality/ cost. This definition may be applicable to the purchase of any item or service, and appears very reasonable. The assessment of health care value based on this definition, however, needs to account for the fact that both quality and cost are to a large extent subjective, especially in the case of chronic diseases when the benefits of the treatment may

L. Balducci $(\bowtie)$

Editor-in-Chief, Moffitt Cancer Center, Tampa, FL, USA

e-mail: lodovico.balducci@moffitt.org be limited and the human cost substantial. I have seen patients declining a treatment that could have kept them alive 6 months longer because they preferred to invest their limited resources in a last trip to their native countries, to greet old friends and mend old wounds. A few years ago, the columns of this journal reported the case of a chief of a dismantled tribe of American Indians who refused chemotherapy for his castrate-resistant prostate cancer [5]. His lifetime goal was to finish writing the history of his tribe and he was concerned that the side effects of chemotherapy might have prevented him from achieving this aim. Every physician has seen patients who requested to be kept breathing beyond any hope of recovery because they had important deadlines to reach or simply because they felt that they owed it to their family and their god. So, one cannot talk about value in medicine without discussing how to assess personal values and how to incorporate personal values in a medical decision.

Given the relevance of the topic, JMP has decided to dedicate two issues to the discussion of value. The second issue will deal with assessment of the value of medical care, while the current issue deals with the assessment of personal values.

Personal values may be defined as the goals or the beliefs that give meaning to a person's life. As such, they are exquisitely personal, cannot be quantified, and influence all important life decisions. As the "case of Peggy Sue" shows, health care professionals cannot deliver effective medicine without understanding a person's values. Peggy Sue's priority was to have a child, and to obtain that goal she was ready to renounce a life-saving bone marrow transplantation for her chronic myelogenous leukemia. Though she had married a physician, she resented a medical establishment that seemed unable to listen to her and help her to achieve her goal. The case of Peggy Sue 
underlines the importance of obtaining a "value history" [6] from a patient whose management may include death and life decisions. Originally, the value history was intended as an instrument to facilitate end-of-life decisions and to avoid a conflict between patient and physician values (the right of a patient to know where a physician stands in terms of abortion and euthanasia, for example). The case of Peggy Sue demonstrates that the scope of a value history is much larger and encompasses all medical decisions.

With globalization, people of different ethnic origins with different personal values emerging from different cultures are likely to come into contact. A practitioner in the modern world needs to be sensitive to these cultural differences in obtaining a value history, as demonstrated by the article by Prof. Surbone and Prof. Baider. The article analyzes the history of a Filipino maid who goes to Israel to take care of a wealthy lady, with whom she establishes a very close relationship, comparable to that of a daughter with her own mother. When the maid develops colon cancer, a conflict develops between the two women that reflects the conflict of two cultures. The maid would prefer to go back to her country despite the limited medical facilities, because the most important thing for her is to die in the care of her family, while her employer insists that she benefit from the advanced Israeli medical system.

The values are best expressed and recognized from the words and metaphors one uses in telling his/her own history. Dr. Elissa Foster, a social scientist specializing in qualitative research, demonstrates how history taking gave the residents of a program of family medicine a much clearer perspective of the patients' values and their own.
These findings highlight the importance of history taking in the delivery of patient-centered medicine, a skill that has been all but forgotten in a technology-oriented medicine.

We had planned to include an article on spiritual and religious value, by Dr. Puchalski, that demonstrates how a religious history may disclose both personal and community resources [7]. The publication of this important work has been delayed to the next issue of JMP due to editorial concerns.

It is always the case in our journal, aware that we have not exhausted the topic of personal values in medicine, we hope we have provided a basis for a healthy discussion with all our readers and empowered our readers to introduce a value history into their busy practices.

\section{References}

1. VanLare JM, Conway PH (2012) Value-based purchasing: national program to move from volume to value. N Engl J Med 26:292-295

2. Conway PH (2009) Values-driven health care: implications for hospital and hospitalists. J Hosp Med 4:507-511

3. Carney PA, Eiff MP, Saultz JW et al (2012) Assessing the impact of Innovative training for family physicians for the patients centered medical homes. J Grad Med Ed 4:16-22

4. Porter ME (2009) A strategy for health care reform-toward a value-based system. N Engl J Med 361:109-112

5. Balducci L (2008) And a time to die. J Med Person 6:99-103

6. Justin R (1987) The value history: a necessary family document. Theor Med 8:275-282

7. Borneman T, Ferrell B, Puchalski CM (2010) Evaluation of the FICA tool for spiritual assessment. J Pain Symptom Manag 40:163-173 